\title{
Kidney involvement in antiphospholipid syndrome - current diagnostic and management problems
}

\author{
Maria Majdan \\ Department of Rheumatology and Connective Tissue Diseases, Medical University, Lublin, Poland
}

Antiphospholipid syndrome (APS) is currently considered a systemic autoimmune disease with coexisting recurrent venous or arterial thrombosis, obstetric complications, and the presence of antiphospholipid antibodies (aPL). The marker antibodies for APS are: anticardiolipin antibodies, anti- $\beta 2$-glikoprotein I antibodies, and lupus anticoagulant (LAC) [1]. APS, which was defined for the first time in 1985, has been diagnosed more and more frequently and is better and better treated. The classification criteria of the disease defined in 2006 include: the presence of venous or arterial thrombosis of any organ, determined obstetric complications, and detection in at least two tests (at least 12 weeks apart) of the marker antiphospholipid antibodies [1]. APS can be isolated (primary APS) in the absence of other autoimmune diseases or (as secondary APS) associated with a number of autoimmune diseases, mostly with systemic lupus erythematosus (SLE).

Many clinical faces of APS may be observed:

- APS with vascular events,

- APS with pregnancy morbidity (OAPS),

- catastrophic antiphospholipid syndrome (CAPS),

- asymptomatic antiphospholipid antibodies,

- APS with non-classification criteria manifestations, like: livedo reticularis, thrombocytopaenia, valvular heart disease, cognitive disorders, APS nephropathy (APSN).

It is recommended that the aforementioned symptoms be included in the classification criteria of APS [1-3].

The classification criteria of the disease defined in 2006 do not include renal involvement, frequently occurring in APS, and mentioned already in the first descriptions of this syndrome [1].

APS causes various pathophysiological effects concerning the kidneys. The renal manifestations of APS may result from thrombosis occurring at any location within the renal vasculature. The renal involvement is associated with both primary and secondary APS [4-10].

There are clinical observations and data from literature showing that kidney involvement is frequently found in APS. There is, however, no clear connection between the presence of a significant level of aPL and advancing of nephropathy, especially occurring in the course of particular connective tissue diseases [8, 11, 12].

The clinical syndromes of renal involvement in APS include [4-10]:

- renal artery stenosis,

- renal vein thrombosis,

- arterial hypertension (from modest to severe),

- antiphospholipid associated nephropathy (APSN) small vessel vaso-occlusive lesions (acute and chronic), - chronic kidney disease at all stages,

- allograft vascular thrombosis.

Large-vessel thrombosis (renal artery stenosis, renal vein thrombosis) represents a well-defined manifestation of APS and can be used for diagnosis of APS. Renal arterial thrombosis is associated with worsening of hypertension, renal infarction with loin pain, and acute or chronic renal failure. Renal vein thrombosis may be presented as proteinuria, decreasing of glomerular filtration rate (GFR), loin pain, haematuria, or secondary pulmonary thrombosis.

APSN, acute or chronic, with variable degrees of severity, and with nephritic or nephrotic syndrome, may be presented in kidney biopsy specimens as thrombotic microangiopathy (TMA), fibrous intimal hyperplasia $(\mathrm{FIH})$, or focal cortical atrophy (FCA) [7, 8, 12].

Recently a new subset of the APS has been proposed: microangiopathic antiphospholipid syndrome (MAPS) comprising those patients presenting thrombotic microangiopathy and antiphospholipid antibodies [5-7].

TMA can be one of the manifestations of the APS and may affect vessels in different organs, resulting in ischaemia and organ failure. The clinical picture depends on the size and type of vessel affected and the organ involved. When capillaries, arterioles, or venules are affected, histopathological characteristics are virtually identical to those of TMA resulting from other caus-

Address for correspondence:

Maria Majdan, Department of Rheumatology and Connective Tissue Diseases, Medical University, Jaczewskiego 8, 20-090 Lublin, Poland, e-mail: maria.majdan@gmail.com

Submitted: 30.10.2017; Accepted: 31.10.2017 
es, such as thrombotic thrombocytopenic purpura and haemolytic-uremic syndrome [5-7].

The true prevalence of APSN is unknown (ranging from 6 to $25 \%$ of patients with APS). An accompanying thrombocytopaenia may limit the possibility of performing the kidney biopsies.

Positive lupus anticoagulant and elevated serum creatinine levels were also identified as significant risk factors for post-biopsy bleeding complications [4-7, 12].

From the few available histopathological assessments of renal biopsy in APS patients, it is known that severe renal histopathological lesions may be found in the absence of significant renal clinical abnormalities [7, 12].

In patients with SLE and secondary APS the clinical presentations of lupus nephritis and antiphospholipid-associated nephropathy are similar, and renal biopsy is necessary to differentiate between these conditions. Recently it has been found that APSN is a severe and of ten unrecognised condition in SLE patients with clinical renal symptoms. There is an increased risk of development of chronic kidney disease and genetic predisposition (HLA-DRB1 alleles) in APSN patients with SLE, compared to patients with lupus nephritis only [12].

Antiphospholipid associated nephropathy is not included in the classification criteria for definite APS. To date, APSN has belonged to non-criteria manifestations of APS. It seems that APSN should be included in the classification criteria for definite APS, together with characteristic clinical and histopathological features [2, 3, 7, 12].

The management of APSN is completely empirical-based. There are no clinical studies of these clinical problems. Like in APS treatment, it is based on: aspirin, heparin, oral anticoagulants, steroids, and immunosuppressive drugs [4, 7-10, 13-15]. The efficient management of hypertension with calcium channel blockers and ACE inhibitors is very important $[7,15]$.

Renal transplantation in patients with APS is very difficult due to the high risk of development of TMA, which is often refractory to aggressive anticoagulation, plasmapheresis, and immunosuppression. Eculizumab, a humanised monoclonal antibody against complement factor 5 , is proposed as salvage therapy. Unfortunately, long-term safety data for eculizumab in complement-mediated TMA are still not available [13-15].

The author declares no conflict of interest.

\section{References}

1. Miyakis S, Lockshin MD, Atsumi T, et al. International consensus statement on an update of the classification criteria for definite antiphospholipid syndrome (APS). J Thromb Haemost 2006; 4: 295-306

2. Gerosa M, Rovelli F. Non-classification criteria w Antiphospholid antibody syndrome - from bench to bedside. In: Antiphospholid antibody syndrome - from bench to bedside, Meroni PL (ed.). Springer, Heidelberg, New York, Dordrecht, London 2015: 61-74.

3. Abreu MM, Danowski A, Wahl DG, et al. The relevance of "non-criteria" clinical manifestations of antiphospholipid syndrome: $14^{\text {th }}$ International Congress on Antiphospholipid Antibodies Technical Task Force Report on Antiphospholipid Syndrome Clinical Features. Autoimmun Rev 2015; 14: 401-414.

4. Gracia-Tello B, Isenberg D. Kidney disease in primary antiphospholipid antibody syndrome. Rheumatology 2017; 56: 1069-1080.

5. Bienaime F, Legendre Ch, Terzi F, et al. Antiphospholipid syndrome and kidney disease. Kidney Int 2017; 91: 34-44.

6. Sciascia S, Baldovino S, Schreiber K, et al. Antiphospholipid syndrome and the Kidney. Semin Nephrol 2015; 35: 478-486.

7. Tektonidou MG. Identification and treatment of APS renal involvement. Lupus 2014; 23: 1276-1278.

8. Alchi B, Griffiths M, Jayne D. What nephrologists need to know about antiphospholipid syndrome. Nephrol Dial Transplant 2010; 25: 3147-3154.

9. Majdan M. The kidney and antiphospholipid syndrome. Pol Arch Med Wewn 2007; 117 (suppl 1): 55-58.

10. Majdan M. Antiphospholipid syndrome and kidney diseases. Pol Merkur Lek 2010; 28: 341-344.

11. Ünlu O, Zuily S, Erkan D. The clinical significance of antiphospholipid antibodies in systemic lupus erythematosus. Eur J Rheumatol 2016; 3: 75-84.

12. Gerhardsson J, Sundelin B, Zickert A, et al. Histological antiphospholipid - associated nephropathy versus lupus nephritis in patients with systemic lupus erythematosus: an observational cross-sectional study with longitudinal follow-up. Arthritis Res Ther 2015; 17: 109.

13. Espinosa G, Cervera R. Current treatment of antiphospholipid syndrome: lights and shadows. Nat Rev Rheumatol 2015; 11 : 586-596.

14. Choi JY, Jung JH, Sung S, et al. Living donor renal transplantation with antiphospholipid syndrome. Medicine 2016; 95 : 46-50.

15. Geethakumari PR, Mille P, Gulati, Nagalla S. Complement inhibition with eculizumab for thrombotic microangiopathy rescues a living-donor kidney transplant in a patients with antiphospholipid antibody syndrome. Transfus Appher Sci 2017; 56: 400-403. 